\title{
Article \\ Symmetric Excitons in an (001)-Based InAs/GaAs Quantum Dot Near Si Dopant for Photon-Pair Entanglement
}

\author{
Xiangjun Shang ${ }^{1,2} \mathbb{D}$, Shulun $\mathrm{Li}^{1,2,3}{ }^{1}$, Hanqing Liu ${ }^{1,2,3}$, Ben Ma ${ }^{1}$, Xiangbin Su ${ }^{1,2,3}$, Yao Chen ${ }^{1}$, Jiaxin Shen ${ }^{1}$, \\ Huiming Hao 1,2,3, Bing Liu ${ }^{4}$, Xiuming Dou 1,2,3, Yang Ji 1,2,3, Baoquan Sun 1,2,3, Haiqiao Ni 1,2,3 \\ and Zhichuan Niu 1,2,3,*
}

check for updates

Citation: Shang, X.; Li, S.; Liu, H.; Ma, B.; Su, X.; Chen, Y.; Shen, J.; Hao, H.; Liu, B.; Dou, X.; et al. Symmetric Excitons in an (001)-Based InAs/GaAs Quantum Dot Near Si Dopant for Photon-Pair Entanglement. Crystals 2021, 11, 1194 https://doi.org/10.3390/ cryst11101194

Academic Editor: Julien Brault

Received: 2 August 2021

Accepted: 24 September 2021

Published: 30 September 2021

Publisher's Note: MDPI stays neutral with regard to jurisdictional claims in published maps and institutional affiliations.

Copyright: (c) 2021 by the authors. Licensee MDPI, Basel, Switzerland This article is an open access article distributed under the terms and conditions of the Creative Commons Attribution (CC BY) license (https:// creativecommons.org/licenses/by/ $4.0 /)$.
1 State Key Laboratory for Superlattice and Microstructures, Institute of Semiconductors, Chinese Academy of Sciences, Beijing 100083, China; xjshang@semi.ac.cn (X.S.); lishulun@semi.ac.cn (S.L.); hqliu@semi.ac.cn (H.L.); lymaben@semi.ac.cn (B.M.); suxb@semi.ac.cn (X.S.); chenyao@semi.ac.cn (Y.C.); shenjx2020@126.com (J.S.); hmhao@semi.ac.cn (H.H.); xmdou04@semi.ac.cn (X.D.); jiyang@semi.ac.cn (Y.J.); bqsun@semi.ac.cn (B.S.); nihq@semi.ac.cn (H.N.)

2 Beijing Academy of Quantum Information Sciences, Beijing 100193, China

3 Center of Materials Science and Optoelectronics Engineering, College of Materials Science and Opto-Electronic Technology, University of Chinese Academy of Sciences, Beijing 100049, China

4 Joint Laboratory of Advanced Semiconductor, Nanjing Guoke Semiconductor Co., Ltd., Nanjing 211800, China; liubing@gksc.ac.cn

* Correspondence: zcniu@semi.ac.cn

\begin{abstract}
The sacrificed-QD-layer method can well control the indium deposition amount to grow InAs quantum dots (QDs) with isotropic geometry. Individual Si dopant above an (001)-based InAs QD proves a new method to build a local electric field to reduce fine structure splitting $(\mathrm{FSS}=\mathrm{X} 1-\mathrm{X} 2)$ and show $\mathrm{D}_{3 \mathrm{~h}}$ symmetric excitons. The lowest FSS obtained is $3.9 \mu \mathrm{eV}$ with the lowest energy $\mathrm{X}$ state (LX) anticlockwise rotate from [1-10] (i.e., zero FSS will be crossed in a proper field). The lateral field projection induces a large eh separation and various FSS, LX, and emission intensity polarization. The lateral field along [1-10] breaks the X1-X2 wavefunction degeneracy for independent $\mathrm{HH}$ and VV cascade emissions with robust polarization correlation. With FSS $\sim 4 \mathrm{eV}$ and $\mathrm{T}_{1} \sim 0.3 \mathrm{~ns}$ fastened in a distributed Bragg reflector cavity, polarization-resolved $X X-X$ cross-correlations show fidelity $\sim 0.55$ to a maximal entangled state $|\mathrm{HH}\rangle+|\mathrm{VV}\rangle$. A higher fidelity and zero FSS will be obtained in the hybrid QD structure with a junction field integrated to tune the FSS and a sub-bandgap excitation to avoid influences from electrons in the barrier.
\end{abstract}

Keywords: single quantum dot; cascade exciton emission; fine structure splitting; local electric field; polarization correlation; emission intensity polarization

\section{Introduction}

Cascade emission in a semiconductor quantum dot (QD) from the biexciton state XX to the ground state $G$ via the intermediate exciton states $X(\mid \uparrow \downarrow$ and $\mid \downarrow \uparrow, \uparrow, \downarrow$ : electron, $\Uparrow, \Downarrow$ : hole spin) can emit polarization-entangled photon pairs ILR > + IRL > (R (L): right (left) circular polarization). However, a real epitaxial QD with in-plane anisotropy (e.g.,

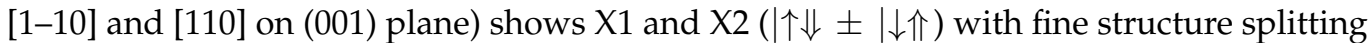
(FSS) and horizontal (H) or vertical (V) linearly polarized two photon emission in a state $\left|\mathrm{HH}>+\mathrm{e}^{\mathrm{iT}}{ }_{1} \mathrm{FSS} / \hbar\right| \mathrm{VV}>\left(\mathrm{T}_{1}\right.$ : time delay between $\mathrm{XX}$ and $\mathrm{X}$ photons, i.e., intrinsic $\mathrm{X}$ radiative lifetime $\mathrm{T}_{\mathrm{X}}$, Figure 1a). The FSS must be smaller than a lifetime-limited radiative linewidth $1 /\left(2 \pi \mathrm{T}_{1}\right)$ (e.g., $4.9 \mu \mathrm{eV}$ for $\mathrm{T}_{1}=134 \mathrm{ps}$ fasten in a microcavity [1]) to erase decay-path information for entanglement [2-4]. For strain-driven (001)-based InAs QDs with a flexible wavelength $(\lambda), 870 \sim 1600 \mathrm{~nm}$ [5-8], structures (e.g., strain-coupled QD bilayer [5], dot-inbarrier [6], or dot-in-well [7]) and microcavity integration, it shows FSS > $10 \mu \mathrm{eV}$ [9-12] typically and spin scattering time $\mathrm{T}_{\mathrm{Ss}} \sim 1.9 \mathrm{~ns}$ [2]. The FSS can be reduced by growing $\mathrm{C}_{3 \mathrm{v}}$ QD on (111) surface [13-17] or a post-grown electric field (different Stark shifts for 
$\mathrm{X} 1$ and $\mathrm{X} 2)[18,19]$ or stress $[10,11,20,21]$ tuning. Due to a deviation of $\mathrm{X} 1$ and $\mathrm{X} 2$ from the main crystal axis [110] and [1-10], the erasure of FSS requires a biaxial stress (inplane) tuning or a combination of stress (in-plane) and electric field (vertical) tuning. Besides, QD geometrical anisotropy (e.g., shape, strain or charged environment) induces light hole (lh)-heavy hole (hh) mixing (in degree $\beta$ ) and photon polarization mixing as the emission intensity polarization (EIP) reflects [22-24], which is quite correlated to the growth parameters such as growth temperature, indium deposition amount and surface migration time. For instance, in our experiments (shown elsewhere), for single InAs QDs at $\lambda=870 \mathrm{~nm}$ formed among dense small QDs at $\lambda=860 \mathrm{~nm}$ (a broad PL spectral profile) by indium migration, a little increase of indium deposition will induce a great EIP transition from a pure dipole to an ellipse and finally a circle (i.e., $\beta \approx 0$ ) with QD emission intensity increasing monotonously. These parameters are well controlled in the sacrificed-QD-layer growth method we developed to obtain QDs with isotropic geometry. In this work, individual Si dopant intentionally added above a QD proves a new method to build a local electric field for electron-hole (eh) separation in the [001] axis to show $\mathrm{D}_{3 \mathrm{~h}}$ symmetric excitons as pyramidal (111) QDs [16,17] and reduce FSS to zero (in an as-grown sample with no fine tuning, the lowest FSS obtained is $3.9 \mu \mathrm{eV}$ with the lowest-energy $X$ state (LX) anticlockwise rotate from [1-10], i.e., zero FSS will be crossed in a proper field). QDs show small lh-hh mixing degree: $\beta \approx 0$ with no doping or in a vertical electric field, and $\beta \approx 0.2$ (close to strain-free GaAs QDs, implying no strain contribution) in an in-plane field. With FSS $\sim 4 \mu \mathrm{eV}$ and T1 $\sim 0.3 \mathrm{~ns}$ in a distributed Bragg reflector (DBR) cavity, polarization-resolved $\mathrm{XX}-\mathrm{X}$ cross-correlations reflect a fidelity $\mathrm{F} \sim 0.55$ to the maximal entangled state $|\mathrm{HH}\rangle+|\mathrm{VV}\rangle$. The lateral field projection in a diverse direction shows a great eh separation for various FSS, LX and EIP. The lateral field along X2 [1-10] breaks $\mathrm{X} 1-\mathrm{X} 2$ wavefunction degeneracy and forbids spin flip between them to show independent $\mathrm{HH}$ and VV cascade emission for polarization-correlated applications. Compared to pyramidal (111) QDs or droplet epitaxial QDs with small FSS as grown, the (001) QDs with Si doping above to reduce FSS have advantages: (1) easy to grow with the growth parameters well controlled; (2) easy to integrate a microcavity (e.g., DBR, circular Bragg grating or photonic crystal) to reduce $\mathrm{T}_{1}$ and enhance QD light extraction; (3) with fewer interface defects. Compared to biaxial stress tuning or the combination of stress and electric field tuning post-grown to reduce FSS [20,21], the local field has advantages: (1) easy to realize and the electric field tuning to erase FSS can be realized by integrating a p-i-n or Shockley junction, facilitating device fabrication; (2) the local field helps to deplete neighbor defects and 'purify' the QD environment; (3) the hybrid QD structure can be well designed (e.g., the field magnitude and the barrier height with AlGaAs [25-27]) and the QD geometry is optimized independently. With a junction field integrated to tune FSS and a sub-bandgap excitation to avoid spin flip from electrons in the barrier, the hybrid QD structure has the potential to get zero FSS and a higher entanglement fidelity.

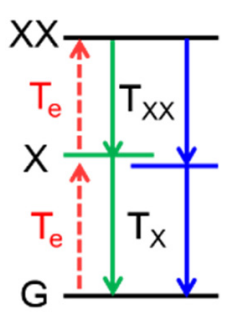

(a)

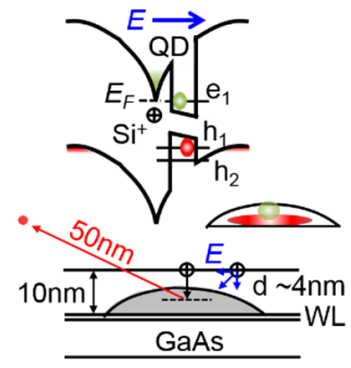

(b)

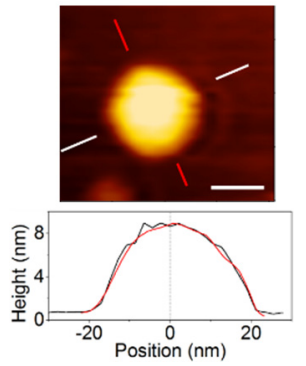

(c)

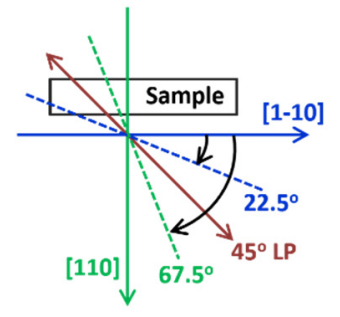

(d)

Figure 1. (a) $X X-X$ cascade emission, green: $H$ [110] and blue: $V$ [1-10], $T_{X X}$ and $T_{X}$ : intrinsic $X X$ and $X$ radiative lifetimes, red dashes: exciton population, $\mathrm{T}_{\mathrm{e}}$ : population time, (b) a QD near a Si dopant, schematic band and epitaxial structures (grey region: QD; circle with '+': Si donor; red dot: a possible defect $>50 \mathrm{~nm}$ away from QD) and electron (green) and hole (red) wavefunctions, (c) AFM image of a typical QD, with symmetric morphological profile (red and black curves) in two orthogonal directions (red and white). Scale bar: $20 \mathrm{~nm}$, (d) element angles for polarization measurement, detailed in the main text. 


\section{Materials and Methods}

Dilute InAs/GaAs QDs were grown by a solid-source molecular beam epitaxy on a semi-insulating GaAs (001) substrate and located at the middle of a planar $1 \lambda$-GaAs cavity between the bottom $(24.5 \times)$ and the top $(4 \times) \mathrm{GaAs} / \mathrm{Al}_{0.9} \mathrm{Ga}_{0.1}$ As DBRs with $\lambda$ at $920 \mathrm{~nm}$ in situ calibrated to enhance light extraction. QDs were grown at a rate of 0.005 monolayer/s in $\mathrm{As}_{4}$ flux pressure of $1 \times 10^{-6}$ Torr at a nominal temperature (T) $540{ }^{\circ} \mathrm{C}$. A sacrificed QD layer was grown first to monitor island by reflection high energy electron diffraction (RHEED) and determine the proper indium deposition amount $[28,29]$ and then evaporated at $670{ }^{\circ} \mathrm{C}$ for $15 \mathrm{~min}$ until the point array in RHEED pattern disappeared (the residual indium atoms were removed). After a $80 \mathrm{~nm} \mathrm{GaAs}$ capping at $580^{\circ} \mathrm{C}$ to flatten the surface and space a possible defect introduced in the sacrificed layer, the formal QDs were grown without substrate rotation to yield gradient indium flux and QD density along [1-10] [30,31]. As the atomic force microscope (AFM) image in Figure 1c shows, the uncapped QD has a height $\sim 8 \mathrm{~nm}$ and a base diameter $\sim 40 \mathrm{~nm}$, symmetric in shape. After a $10 \mathrm{~nm}$ GaAs capping, a Si $\delta$-doping layer in surface density of $1 \times 10^{8} \mathrm{~cm}^{-2}$ (i.e., individual $\mathrm{Si}$ atoms in a microregion) was added to compensate the background p-type impurities (e.g., carbons, that induce a dominant $\left.X^{+}[32,33]\right)$ for dominant $X$ and $X X$ emissions, followed by a $20 \mathrm{~nm} \mathrm{GaAs}$ before raising $\mathrm{T}$ to $580^{\circ} \mathrm{C}$ for the residual structure growth. See Figure $1 \mathrm{~b}$, the $10 \mathrm{~nm}$ GaAs capping built a 4-nm space $(d)$ between the QD and the Si dopant above it, corresponding to the maximal Coulomb field of $E=e / 4 \pi \varepsilon_{0} \varepsilon_{r} \mathrm{GaAs}_{\mathrm{As}} d^{2}=75 \mathrm{kV} / \mathrm{cm}$ at the QD to reduce electron (at QD top) and hole (at QD base) wavefunction overlap [34] for small FSS and higher $\mathrm{D}_{3 \mathrm{~h}}$ exciton symmetry, i.e., a three-fold rotation symmetry and a mirror one along the [001] axis. The local field helps to purify the QD environment from fluctuation charges of a hole defect [35] (red dot) that is at least $50 \mathrm{~nm}$ away from the hybrid QD structure and only induces a linewidth broadening of $\sim 15 \mu \mathrm{eV}$ from spectral diffusion. The influence is only from electrons near the $\mathrm{Si}$ dopant for tunneling and spin flip. For a $\mathrm{Si}$ dopant near a QD, the extra electron fills in QD with lower energy level and builds a local electric field and a Fermi level pin at QD level in favor of XX population. These electrons are depleted by filling neighbor defects to enhance the local field and show a dominant $\mathrm{X}^{+}$and a weak $\mathrm{XX}$ with a strong electron tunneling (the $\mathrm{XX}$ population is unaffected in the sub-bandgap excitation). The $4 \mathrm{~nm}$ barrier (i.e., height and thickness) is flexible for design to optimize the local field and reduce tunneling [19]. Similarly, in a two-dimensional heterostructure, modulated doping to form electron gas is inserted in the AlGaAs barrier a little spaced from the interface. Since the $\mathrm{Si}$ atoms are randomly distributed, to build a desired vertical field, one dopant for each QD is desired. Molecular beam epitaxy is quite suitable for the control of such QD/barrier interface and the growth of such hybrid QD structure. Here, since the local field was diverse and unknown in an as-grown sample, to investigate the field dependence, no AlGaAs capping was used and a high field was evidenced from a weak XX and an electron level coupling between QD $e_{1}$ and Rydberg level at Si dopant.

Single QD spectroscopy was performed by a micro-photoluminescence $(\mu \mathrm{PL})$ setup with fiber routes and a $0.75 \mathrm{~m}$ grating monochromator equipped with a liquid-nitrogen cooled charge coupled device camera (spectral resolution: $50 \mu \mathrm{eV}$ ). The sample cooled in a helium-flow cryostat $(\mathrm{T}=5 \mathrm{~K})$ was $\mathrm{cw}$-excited at $632.8 \mathrm{~nm}$ [32]. A polarization selective filtering (i.e., a half-wave plate (HWP) and a linear polarizer (LP)) suppresses a distinct fine-structure component to observe the subtle shift below the spectrometer resolution in resolution of $\sim 2 \mu \mathrm{eV}$. As Figure 1d indicates, the sample strip along [1-10] was positioned as reference, the LP was fixed at $45^{\circ}$ from [1-10] while the HWP (dash lines) rotated clockwise with angle $\alpha$ to select a linear polarization: $\alpha=67.5^{\circ}$ for that along [110] and $\alpha=22.5^{\circ}$ for that along [1-10]. X and XX showed FSS oscillations in the same scale and opposite signs; $\mathrm{X}^{+}$and $\mathrm{X}^{-}$showed zero FSS. The electron level coupling between QD e1 and Rydberg level at Si dopant in a high field induced FSS in $\mathrm{X}^{-}$(1e1h in QD and 1e near Si dopant) and zero FSS in XX (1e2h in QD and 1e near Si dopant). The FSS of each QD was characterized to find one $<7 \mu \mathrm{eV}(30 \%)$ and $\sim 4 \mu \mathrm{eV}$ (only 10\%). For as-grown sample 
with no fine tuning, the FFS and LX polarization (i.e., angle $\theta$ from [110]) were strongly dependent on the local field. The various FSS and LX $\theta$ can be well depicted by X1-X2 coupling [19]: in a vertical field, for anti-crossing coupling, as the field increased, the FSS first reduced to a minimum $\Delta$ and then increased; the LX $\theta$ varied slowly from $\sim 90^{\circ}$ (X2 along [1-10]) in a low field to $0^{\circ}<\theta<90^{\circ}$ in a medium field to get $\Delta$ and $\sim 0^{\circ}$ (X1 along [110]) in a much higher field; for crossing coupling with zero $\Delta$, a fast anticlockwise rotation from [1-10] $\theta=-90^{\circ}$, equivalent to $\theta>90^{\circ}$, occurred, as shown in Figures 2 and 3; for an in-plane field, the case was opposite: clockwise rotation corresponds to a small FSS and anticlockwise rotation a large FSS, as shown in Figure 4. The EIP (i.e., angle $\varphi$ from [110]) was orthogonal to the LX that reflected a stronger emission in the higher-energy $\mathrm{X}$ state (HX) from lh-hh mixing [22] in the local field; there was little strain contribution since a QD with no doping shows nearly isotropic emission intensity (e.g., QD29 and 30 in Figure 4). The EIP was obvious in a lateral field and disappeared in a high vertical field; its degree (i.e., $\beta$ ) characterized the geometrical anisotropy of QDs buried in GaAs, identical in the same sample (e.g., QD20, 27 and 28 in Figures 3 and $4 \mathrm{~b}$ with a $12 \mathrm{~nm}$ capping before $\mathrm{Si}$ dopant). Polarization-resolved $X X-X$ cross-correlations $g^{2} x x_{i} x_{j}$ in the basis $\{i, j\}=\{H, V$, R, L, D (diagonal), A (antidiagonal)\} were measured by a Hanbury Brown-Twiss setup integrated on the uPL [32] in time resolution $\sim 600 \mathrm{ps}$, including a 50:50 beamsplitter, each beam with a narrowline bandpass (NBP, $<0.5 \mathrm{~nm}, \mathrm{OD} 6)$ to filter $\mathrm{X}$ or $\mathrm{XX}$ photons and a polarization filter (i.e., an LP and a quarter-wave plate (QWP), for $\mathrm{H} / \mathrm{V}$ and $\mathrm{D} / \mathrm{A}$, only LP in different angles was used; for R/L, LP and QWP with $45^{\circ}$ angle offset were used) before fiber collection, two avalanched $\mathrm{Si}$ single-photon counters and a time-to-digital converter for coincidence count histogram. Polarization correlation and entanglement fidelity $\mathrm{F}$ were reflected in the correlation contrasts $C_{i j}=\left(g^{2}{ }_{i i}-g^{2}{ }_{i j}\right) /\left(g^{2}{ }_{i i}+g^{2}{ }_{i j}\right)(j$ opposite to $i)$, and compared to the theoretical (F, FSS) fitting curve $[2,3]$.

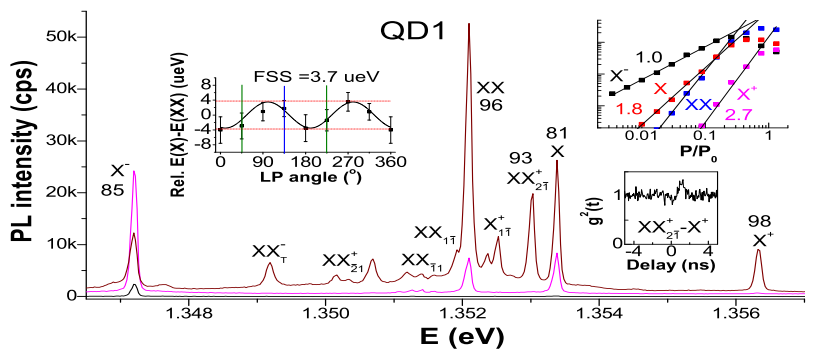

(a)

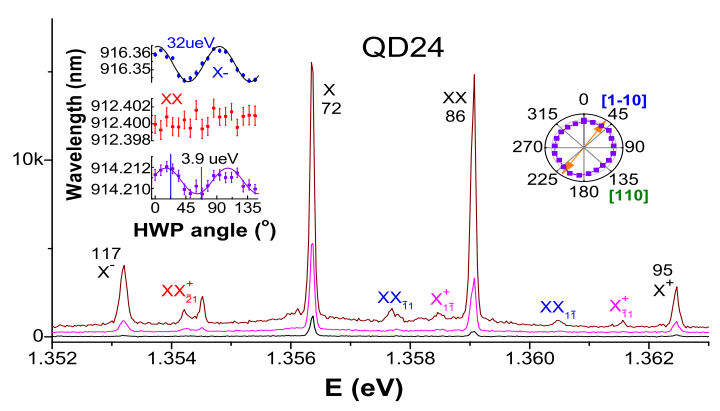

(c)

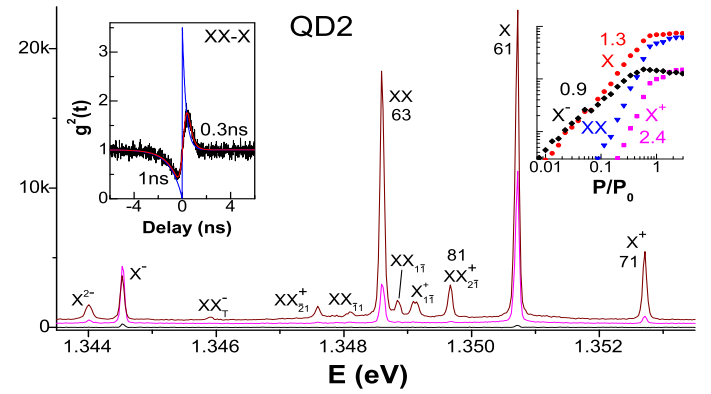

(b)

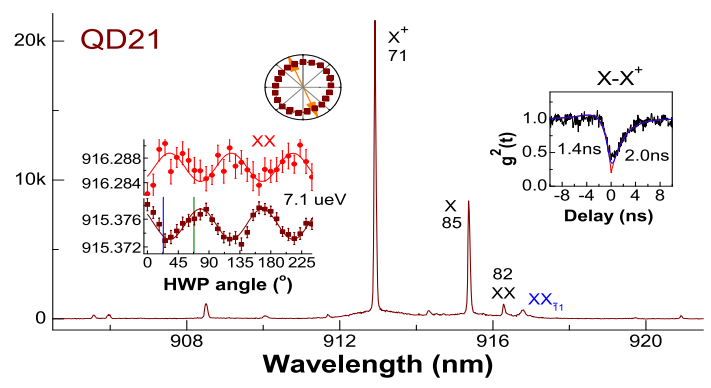

(d)

Figure 2. Excitation power (black: weak, pink: moderate, wine: high)-dependent $\mu \mathrm{PL}$ spectra of three QDs with $\mathrm{D}_{3 \mathrm{~h}}$ symmetric $\mathrm{XX}_{21}{ }^{+}, \mathrm{XX}_{11}$ and $\mathrm{X}_{11}{ }^{+}$exciton emissions, (a) QD1; (b) QD2; (c) QD24, and (d) QD21 (in a high field like QD24 and with a dominant $\mathrm{X}^{+}$). The numbers near exciton labels represent spectral linewidths. Insets: intensity excitation power 
dependence (slope marked, $\mathrm{P}_{0}=25 \mu \mathrm{W}$ ) in (a) QD1 and (b) QD2; photon cross-correlations of $\mathrm{XX}_{2 \overline{\mathrm{i}}}{ }^{+}-\mathrm{X}^{+}$cascade in (a) QD1, XX-X cascade in (b) QD2, and $X-X^{+}$in (d) QD21 (fitting times are given); polarization-resolved FSS oscillations in (a) QD1 (FSS $=3.7 \mu \mathrm{eV}$, measured by a rotate LP only $\left(45^{\circ}\right.$ corresponds to [110]) and extracted from a sinusoid fitting of the polarization-related $X X-X$ relative energy offset), (c) QD24 (FSS $=3.9 \mu \mathrm{eV}$ in $\mathrm{X}, 0$ in $\mathrm{XX}$ and $32 \mu \mathrm{eV}$ in $\mathrm{X}^{-}$) and (d) QD21 (FSS = 7.1 $\mu \mathrm{eV}$ in both $\mathrm{X}$ and $\mathrm{XX}$ ); EIP in a polar plot (orange arrow indicates the LX orientation); green line: along [110] with $\alpha=67.5^{\circ}\left(135^{\circ}\right.$ in polar plot), blue line: along [1-10] with $\alpha=22.5^{\circ}$.

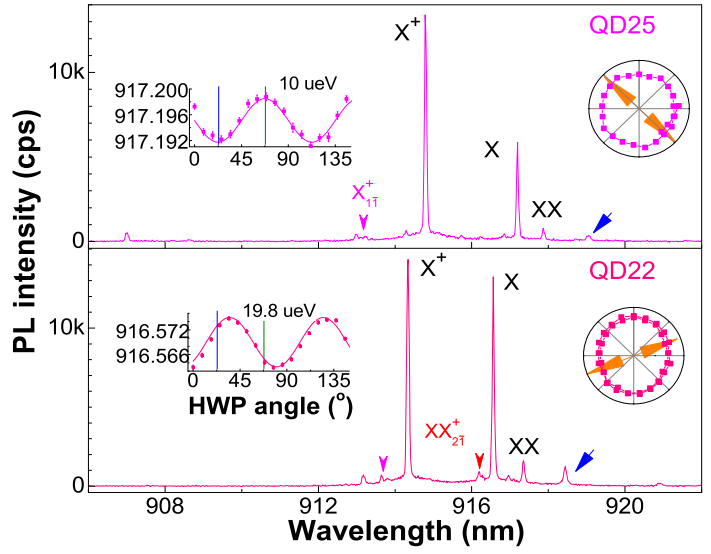

(a)

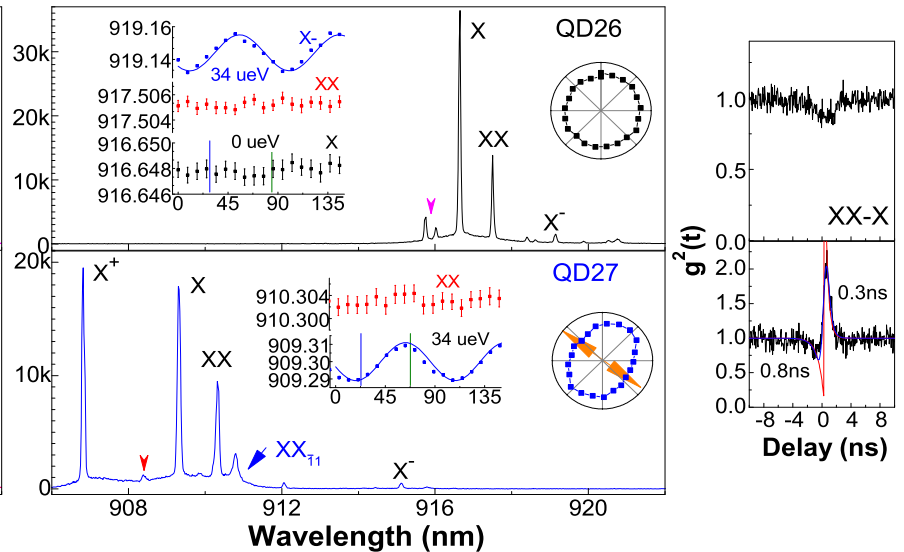

(b)

Figure 3. (a) $\mu \mathrm{PL}$ spectra, FSS oscillation with fitting and EIP in polar plot (orange arrow indicates LX) of QD22 and QD25 27 in a strong vertical field with a dominant $\mathrm{X}^{+} ;(\mathbf{b}) \mathrm{XX}-\mathrm{X}$ correlations in QD26 and QD27 (fitting times are given).

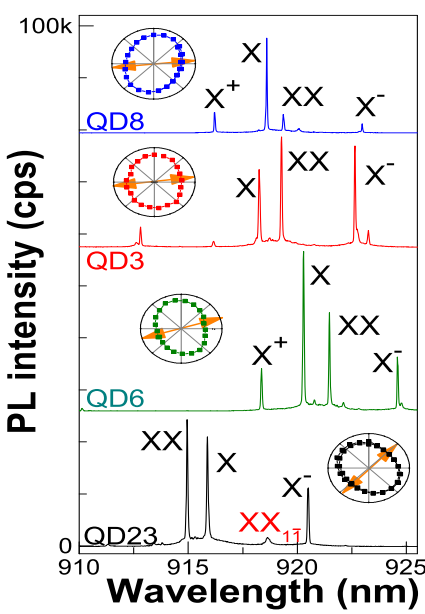

(a)

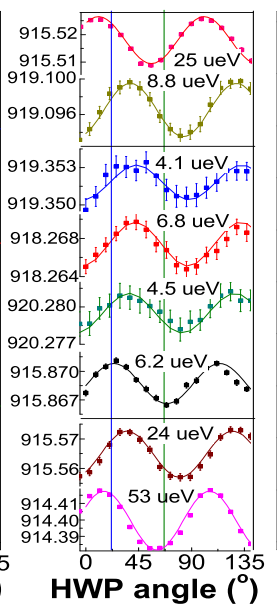

(b)

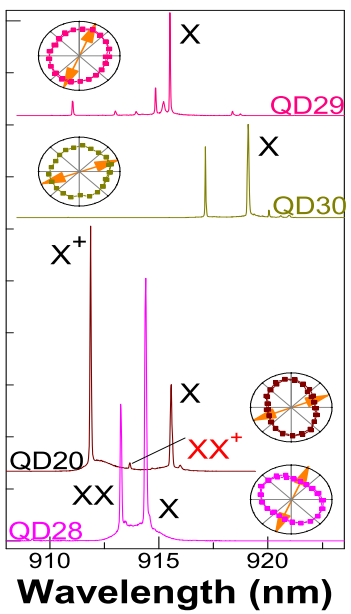

(c)

Figure 4. $\mu \mathrm{PL}$ spectra and EIP (arrow indicates LX) of (a) left: QD23, QD6, QD3 and QD8 with a 10-nm capping and Si dopant varied from the center for a larger lateral field and (c) right: QD30 and QD29 un-doped, QD20 and QD28 with a 12-nm capping before dense Si dopant; (b) middle: their FSS oscillations with sinusoid fitting, blue: [1-10], green: [110]. Each QD is in a different color for clarity.

\section{Results and Discussion}

\section{1. $D_{3 h}$ Symmetric Exciton Emissions}

Figure 2 presents the QDs with $\mathrm{D}_{3 \mathrm{~h}}$ symmetric exciton emissions in a spectral feature as pyramidal (111) QDs [16,17] from valence band mixing and $\mathrm{lh}_{2} \mathrm{~h}_{2}$ for $\mathrm{e}_{1}-\mathrm{h}_{2}$ transitions $\mathrm{XX}_{2 \overline{1}}{ }^{+}, \mathrm{X}_{1 \overline{1}}{ }^{+}$and $X X_{1 \overline{1}}$ and $\mathrm{e}_{1}-\mathrm{h}_{1}$ ones $\mathrm{XX}_{\overline{2} 1^{\prime}}^{+}, \mathrm{X}_{\overline{1} 1}{ }^{+}$and $\mathrm{XX}_{\overline{1} 1}$ (indexes mean the hole numbers in $h_{1}$ (first) and $h_{2}$ (second) with a bar classifying the transitions) $[16,33]$, located around $\mathrm{XX}$ as a mirror. $\mathrm{XX}_{2 \overline{1}}{ }^{+}$singlet built a cascade with $\mathrm{X}^{+}$as the bunching peak in their cross- 
correlation reflects, weaker than before [33] due to a fast electron capture in $\mathrm{X}^{+}$to populate $\mathrm{XX} ; \mathrm{X}_{1 \overline{\mathrm{i}}}{ }^{+}$doublet (split by $\Delta_{\mathrm{hh}} \pm\left(\Delta^{\mathrm{I}} \mathrm{eh}+\Delta^{\mathrm{II}} \mathrm{eh}^{\mathrm{h}}\right): 150 \mu \mathrm{eV}$ in QD1 or $50 \mu \mathrm{eV}$ in QD2) and $\mathrm{XX}_{1 \overline{1}}$ doublet (split by $\Delta_{\mathrm{hh}}-\Delta^{0}{ }_{\mathrm{eh}}$ [16] of near zero); a broad $\mathrm{XX}_{\mathrm{T}}{ }^{-}$(doublet split by $\sim \Delta^{0}{ }_{\mathrm{eh}} / 2$ [33]) also appeared. In QD1, the three peaks in $X X_{\overline{1} 1}$ were well depicted by $D_{3 h}$ transition diagram ( $\sigma$-polarized) while the three peaks in $X X_{\overline{2} 1}^{+}$with the $v_{\overline{i i i}}$ branch appearing to reflect $C_{3 v}$ [16], the lack of a mirror symmetry; in $Q D 2$, both $X X_{\overline{2} 1}^{+}$and $X X_{\overline{1} 1}$ showed one peak from $\mathrm{D}_{3 \mathrm{~h}}$ (z-polarized) [16]. In QD24, the higher field showed a relatively lower emission intensity and made these peaks a little far from XX that has a negative binding energy. QD5 in Supplementary Materials (SM) shows more $\mathrm{D}_{3 \mathrm{~h}}$ exciton emissions while the QDs in a high electric field, e.g., QD21 and QDs in Figures 3 and $4 a$, only show $\mathrm{e}_{1}-\mathrm{h}_{2}$ ones, $\mathrm{XX}_{2 \overline{\mathrm{i}}}{ }^{+}$singlet located between $\mathrm{X}$ and $\mathrm{XX}, \mathrm{XX}_{1 \overline{\mathrm{i}}}$ doublet split by $\Delta_{\mathrm{hh}}-\Delta^{0}{ }_{\mathrm{eh}}$ of $70 \mu \mathrm{eV}$ and $\mathrm{X}_{1 \mathrm{i}}{ }^{+}$doublet far from $\mathrm{XX}$, from a large $\mathrm{h}_{1}-\mathrm{h}_{2}$ energy offset in the field. For epitaxial QDs with a less lateral confinement, the eh separation and Stark shift in the lateral direction to tune FSS requires a relatively lower electric field [36]. The appearance of the $D_{3 h}$ symmetric excitons reflects a vertical field for eh separation along [001] for higher exciton symmetry; the FSS, varying from dot to dot, e.g., QD4 in SM with similar spectral features and a high $\mathrm{XX}$ as QD1 but FSS of $11 \mu \mathrm{eV}$, is from an in-plane field projection as a perturbation; the FSS is also reported in pyramidal QDs from composition alloy or piezoelectric potential [15]. For QD1 and QD2, the Si dopant filled two electrons in QD e (Fermi level pin), resulting in the first appearance of $X^{-}$(slope: 1.0 or 0.9 ) before $X$ under weak excitation (black line), a higher slope for $\mathrm{X}$ (1.8 or 1.3) and an effective $\mathrm{XX}$ population under high excitation (wine line) in the slope of 2.4 2.7, the same as $\mathrm{X}^{+}$(2h capture); QD1 had a higher total intensity and a relatively higher $\mathrm{XX}$ saturated intensity ( $50 \mathrm{kcps})$, reflecting a fluent electron capture due to electron level coupling by tunneling that also broadens the spectral line. The line-shape of each spectral line is a Gaussian envelope with different linewidths, broadened by $10 \sim 70 \mu \mathrm{eV}$ after subtracting the spectrometer resolution, and tails in the lower-energy side reflecting wavefunction tunneling into the barrier, in spite of a natural radiative linewidth of a few $\mathrm{ueV}$ in a Lorentzian line-shape. The spectral diffusion from fluctuating charges $>50 \mathrm{~nm}$ from a QD leads to a linewidth broadening $\sim 15 \mu \mathrm{eV}$ (Stark shift slope of $\sim 50 \mu \mathrm{eV} /(\mathrm{kV} / \mathrm{cm})$ [18]); the rest broadening of $0 \sim 60 \mu \mathrm{eV}$ is from electron tunneling and wavefunction coupling between $\mathrm{QD} \mathrm{e}_{1}$ and Rydberg level at the Si dopant. In QD2, $\mathrm{XX}_{2 \mathrm{i}}{ }^{+}$(linewidth: $\left.81 \mu \mathrm{eV}\right)$ with respect to $\mathrm{X}^{+}(71 \mu \mathrm{eV}), \mathrm{X}(61 \mu \mathrm{eV}), \mathrm{XX}(63 \mu \mathrm{eV})$ and $\mathrm{X}^{-}(62 \mu \mathrm{eV})$ reflected no electron tunneling broadening; in $\mathrm{QD} 1, \mathrm{XX}_{2 \overline{1}}{ }^{+}(93 \mu \mathrm{eV})$ with respect to $\mathrm{X}^{+}(98 \mu \mathrm{eV}), \mathrm{X}(81 \mu \mathrm{eV}), \mathrm{XX}(96 \mu \mathrm{eV})$ and $\mathrm{X}^{-}(85 \mu \mathrm{eV})$ reflected an electron tunneling broadening of $\sim 45 \mu \mathrm{eV}$ for $\mathrm{XX}$ and $\mathrm{X}^{+}$that showed the same linewidth (similar for QD4 in SM). As a result, in QD1 and QD4, the XX population at saturation was greatly enhanced. The QDs in Figure 4a also showed the first appearance of $\mathrm{X}^{-}$, a relatively higher XX and LX clockwise rotate from [1-10] related to a lateral field projection. In contrast, for QD24, the Si dopant built a strong vertical field for small FSS of $3.9 \mu \mathrm{eV}$ in $X$; electron level coupling in the field induces abnormal FSS, zero in XX and $32 \mu \mathrm{eV}$ in $\mathrm{X}^{-}$and $\mathrm{X}^{+}$, a less electron tunneling broadening of $\sim 16 \mu \mathrm{eV}$ for $\mathrm{XX}$ (linewidth: $86 \mu \mathrm{eV}$ ) and $\mathrm{X}^{-}(117 \mu \mathrm{eV})$ after subtracting its FSS, but zero for $\mathrm{X}^{+}(95 \mu \mathrm{eV})$ after subtracting its FSS due to its greatly lower field ( $2 \mathrm{~h}$ in QD and 1e at Si dopant) and the first appearance of $X$ under weak excitation. Mostly, a QD in a vertical field with little tunneling shows a dominant $\mathrm{X}^{+}$and a weak XX (e.g., QD21), with the same FSS for $\mathrm{X}$ and XX. The XX-X cross-correlation in QD2 shows an asymmetric profile, with characteristic times for the antibunching dip of $1 \mathrm{~ns}$ (i.e., $2 \mathrm{~T}_{\mathrm{e}}$ for $\mathrm{XX}$ population and $\mathrm{T}_{\mathrm{e}}=0.5 \mathrm{~ns}$ for $\mathrm{X}$ ) and the bunching peak of $T_{1} \sim 0.3$ ns, i.e., intrinsic $X$ radiative lifetime, the $X X$ repopulation (i.e., the upper $T_{e}$ in Figure 1a) therefore competes with the cascade emission. The bunching peak remains if XX has tunneling (zero FSS) while it disappears if X has tunneling (zero FSS), see QD26 and QD27 in Figure 3. An asymmetric antibunching profile is shown in $\mathrm{X}-\mathrm{X}^{+}$cross-correlation in QD21 with the fitting times $1.4 \mathrm{~ns}$ reflecting slow electron capture and $2.0 \mathrm{~ns}$ reflecting $\mathrm{X}^{+}(1 \mathrm{e} 2 \mathrm{~h})$ population. The different correlation profiles help to identify the dominant $\mathrm{X}, \mathrm{X}^{+}$ or XX peak. 


\subsection{FSS Field Dependence}

The FSS field dependence is illustrated in Figure 3 (QDs in a high vertical field with a dominant $\mathrm{X}^{+}$and a weak XX) and Figure 4 (QDs with a lateral field (Figure 4a: QDs with $10 \mathrm{~nm}$ GaAs capping before Si dopant and lateral field projection for EIP; Figure 4b: QD20 and QD28 with $12 \mathrm{~nm}$ capping and dense Si dopants $\left(5 \times 10^{8} \mathrm{~cm}^{-2}\right)$ for a diverse lateral field; QD29 and QD30 undoped with an intrinsic weak in-plane field (e.g., strain or charged environment) to show LX varying from X2 [1-10]). For a vertical field, the FSS is tuned in a scheme: LX clockwise rotation from [1-10] shows a large FSS, $19.8 \mu \mathrm{eV}$ in QD22 and $10 \mu \mathrm{eV}$ in QD25, i.e., $\mathrm{X} 1-\mathrm{X} 2$ anti-crossing; $\mathrm{LX}$ anticlockwise rotation with $\theta=103^{\circ}\left(158^{\circ}\right)$ in QD24 (QD21) shows a small FSS, $3.9 \mu \mathrm{eV}(7.1 \mu \mathrm{eV})$, i.e., $\mathrm{X} 1-\mathrm{X} 2$ crossing with zero $\Delta$. For a lateral field, the FSS is tuned in an opposite scheme: LX clockwise rotation from [1-10] shows a small FSS, 4 7 $\mu \mathrm{eV}$ in QD3, 6, 8 and 23 and $24 \mu \mathrm{eV}$ in QD20 (a QD with similar EIP and LX as QD20, QD19 in SM, shows FSS $=5.9 \mu \mathrm{eV}$ ), LX anticlockwise rotation shows a large FSS,

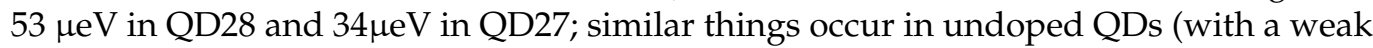
lateral field (e.g., strain or charged environment) rotates LX from [1-10]): $8.8 \mu \mathrm{eV}$ in QD30 with LX clockwise rotate and $25 \mu \mathrm{eV}$ in QD29 with LX anticlockwise rotate. The electron tunneling in XX (zero FSS) improves XX population in QD27 and shows a smaller $2 \mathrm{~T}_{\mathrm{e}}$ of $0.8 \mathrm{~ns}$ in $\mathrm{XX}-\mathrm{X}$ correlation and the $\mathrm{T}_{1}$ keeps $0.3 \mathrm{~ns}$. QD26 even showed electron tunneling in $X$ for zero FSS and no cascade emission (i.e., no bunching in XX-X correlation). With a high barrier to avoid tunneling and a sub-bandgap excitation, the $X X$ population will keep dominant. QD27, 28 and 20 are QDs with a larger GaAs capping $(12 \mathrm{~nm})$ before a higher Si doping $\left(5 \times 10^{8} \mathrm{~cm}^{-2}\right)$ that show a strong lateral field to cause the obvious $\operatorname{EIP}(\beta \approx 0.2)$, a $C_{2 \mathrm{v}}$-featured $\mathrm{XX}^{+}$peak (QD20) andD $\mathrm{D}_{3 \mathrm{~h}}$ symmetric $\mathrm{XX}_{\overline{\mathrm{i}}}$ peak (only in QD27 in a much strong vertical field with LX clockwise rotation to nearly along [110] and FSS $=34 \mu \mathrm{eV}$ far beyond the minimum). Compared to it, QD25 with LX nearly along [110] showed a smaller FSS $(10 \mu \mathrm{eV})$ reflecting a fast LX rotation related to its high symmetry, a little misaligned from the main crystal axis [1-10]. QD22 and QD25 showed nonzero FSS in XX (not presented) the same as QD21. QD24 in Figure 2 and QD22, 25 and 26 in Figure 3 in a high vertical field showed zero EIP; while QDs with a lateral field projection (QDs in Figure $4 a)$ showed an obvious EIP $(\beta<0.2)$ in different orientations, consistent with the EIP-electric field dependence as reported [37]. QD30 and QD29 with no doping showed no EIP. QD20, 27 and 28 showed a large EIP $(\beta=0.2)$ related to the growth parameters.

The LX $\theta$ and EIP orientation (i.e., angle $\varphi$ from [110]), as summarized in Table 1, were nearly orthogonal, reflecting a stronger emission in the HX; in a dipole emitter assumption, the EIP orientation reflects the lateral field direction. The lateral field along HX increases its hh energy to mix the LX lh (in spite of lh-hh separation of tens of meV) and reduce transition to the LX hh [22]. In QD23 with LX along [1-10], the lateral field along HX [110] increased its Stark shift for a small FSS = HX-LX $(6.2 \mu \mathrm{eV})$ and XX showed a negative binding energy (similar for QD28); in QD3, 6 and 8, the LX was rotate clockwise from [1-10] $\left(\theta\right.$ of $\left.50 \sim 60^{\circ}\right)$ for a smaller FSS $(4.1 \sim 6.8 \mu \mathrm{eV})$; the lateral field is in a direction as the $\varphi$ $\left(-60 \sim-20^{\circ}\right)$ depicted; in QD7 in Figure 5, the lateral field was along LX [1-10] to increase its Stark shift for a large FSS $(22 \mu \mathrm{eV})$ and break wavefunction degeneracy. 
Table 1. PA $\varphi$, LX $\theta$ and FSS for QDs. Sample A: with $10 \mathrm{~nm}$ GaAs capping before Si doping in density of $1 \times 10^{8} \mathrm{~cm}^{-2}$; Sample B: with $12 \mathrm{~nm}$ capping before Si doping in density of $5 \times 10^{8} \mathrm{~cm}^{-2}$; Sample C: with no doping. QD21, 22, 24 27: in a high vertical field; QD3, 6, 7, 8, 23 and QD19, 20, 28: with a lateral field projection.

\begin{tabular}{cccccccccc}
\hline Sample & QD & PA $\varphi$ & LX $\theta$ & FSS & Sample & $\begin{array}{c}\text { QD } \\
\text { No. }\end{array}$ & PA $\boldsymbol{\text { LX } \theta}$ & FSS \\
\hline A & QD24 & no & $103^{\circ}$ & $3.9 \mu \mathrm{eV}$ & A & QD25 & no & $0^{\circ}$ & $10 \mu \mathrm{eV}$ \\
A & QD21 & $105^{\circ}$ & $158^{\circ}$ & $7.1 \mu \mathrm{eV}$ & A & QD26 & no & - & $0 \mu \mathrm{eV}$ \\
A & QD22 & $135^{\circ}$ & $60^{\circ}$ & $19.8 \mu \mathrm{eV}$ & B & QD27 & $100^{\circ}$ & $12^{\circ}$ & $34 \mu \mathrm{eV}$ \\
\hline A & QD8 & $-60^{\circ}$ & $50^{\circ}$ & $4.1 \mu \mathrm{eV}$ & B & QD20 & $-25^{\circ}$ & $65^{\circ}$ & $24 \mu \mathrm{eV}$ \\
A & QD3 & $-45^{\circ}$ & $50^{\circ}$ & $6.8 \mu \mathrm{eV}$ & B & QD19 & $-20^{\circ}$ & $55^{\circ}$ & $5.9 \mu \mathrm{eV}$ \\
A & QD6 & $-20^{\circ}$ & $60^{\circ}$ & $4.5 \mu \mathrm{eV}$ & B & QD28 & $20^{\circ}$ & $110^{\circ}$ & $53 \mu \mathrm{eV}$ \\
\hline A & QD23 & $0^{\circ}$ & $90^{\circ}$ & $6.2 \mu \mathrm{eV}$ & C & QD30 & no & $65^{\circ}$ & $8.8 \mu \mathrm{eV}$ \\
A & QD7 & $90^{\circ}$ & $110^{\circ}$ & $22 \mu \mathrm{eV}$ & C & QD29 & $95^{\circ}$ & $110^{\circ}$ & $25 \mu \mathrm{eV}$ \\
\hline
\end{tabular}

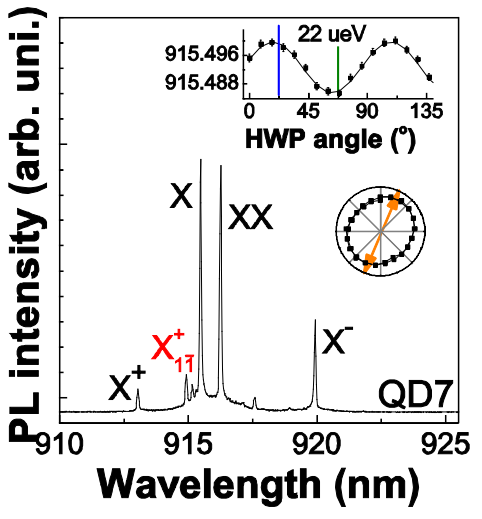

(a)

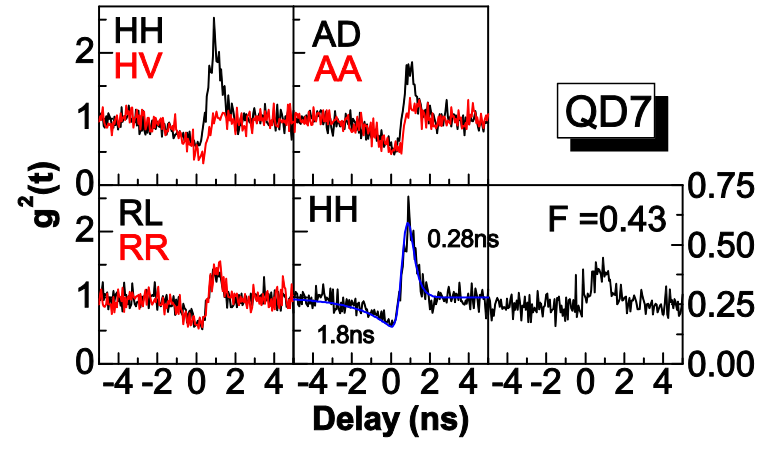

(b)

Figure 5. QD7 with EIP along [1-10]. (a) $\mu \mathrm{PL}$ spectrum, FSS oscillation and EIP in polar plot (orange arrow indicates LX); (b) polarization-resolved XX-X correlations.

\section{3. $X X-X$ Photon-Pair Correlations}

Figure 6 presents the measured polarization-resolved $X X-X$ correlations in QDs with dominant $X$ and $X X$ emissions. A negative contrast in the RL/RR basis and a positive one in the $\mathrm{HH} / \mathrm{HV}$ and $\mathrm{AD} / \mathrm{AA}$ bases indicate the maximal entangled state $|\mathrm{HH}\rangle+|\mathrm{VV}\rangle$ and $\mathrm{F}=\left(1+\mathrm{C}_{\mathrm{HV}}+\mathrm{C}_{\mathrm{DA}}-\mathrm{C}_{\mathrm{RL}}\right) / 4$ to it: $\mathrm{F}=0.55$ in QD6 $(\mathrm{FSS}=4.5 \mu \mathrm{eV}), \mathrm{F}=0.48$ in QD23 $(6.2 \mu \mathrm{eV})$; both $\mathrm{HH}$ and $\mathrm{HV}$ bases show bunching, a little from spin precession in time h/FSS [3] $(0.6 \mathrm{~ns}$ for FSS $=6 \mu \mathrm{eV})$ and mainly from spin flip with electrons in the barrier; a large correlation contrast remains in the AA/AD basis; in QD6 with a smaller FSS, the correlation contrast in the RR/RL basis is large (0.49), reflecting a nice restoring of X1-X2 degeneracy. QD8 and QD3 show a negative contrast in the AA/AD basis and a positive one in the $\mathrm{RL} / \mathrm{RR}$ basis, reflecting $\mathrm{F}=\left(1+\mathrm{C}_{\mathrm{HV}}-\mathrm{C}_{\mathrm{DA}}+\mathrm{C}_{\mathrm{RL}}\right) / 4$ to the maximal entangled state $|\mathrm{HH}>-| \mathrm{VV}>: \mathrm{F}=0.57$ in QD8 $(\mathrm{FSS}=4.1 \mu \mathrm{eV})$ and $\mathrm{F}=0.49$ in QD3 $(\mathrm{FSS}=6.8 \mu \mathrm{eV})$, with a large contrast in the $\mathrm{HH} / \mathrm{HV}$ basis $(0.73$ in QD8, 0.44 in QD3, in contrast to 0.21 in QD23 with nearly the same FSS as QD3) and a nearly zero contrast in the RR/RL basis since the lateral field has a large component on [1-10] to break X1-X2 wavefunction degeneracy and block spin flip between them, i.e., $\mathrm{HH}$ and VV cascade emissions are independent. This phenomenon is also clearly shown in QD7 in Figure 5, where the EIP (i.e., lateral field) was completely along [1-10] and the LX was nearly parallel to it for a large Stark shift, a large FSS and a large eh separation to break X1-X2 wavefunction degeneracy; like QD8, the correlation contrast in the $\mathrm{HH} / \mathrm{HV}$ basis was 0.46 even for $\mathrm{FSS}=22 \mu \mathrm{eV}$; the correlation contrast in the RR/RL basis was zero, reflecting independent $\mathrm{HH}$ and VV cascade emissions. There was no entanglement, while the robust $\mathrm{HH} / \mathrm{VV}$ polarization correlations in this kind of QDs, insensitive on the FSS, can be used as photon-pair sources for quantum detection. The weak XX in QD8 was equivalent to a pulsed excitation of 
$\mathrm{XX}-\mathrm{X}$ cascade emission to avoid $\mathrm{XX}$ repopulation for the highest $\mathrm{HH}$ bunching peak; it also showed a larger $\mathrm{T}_{1} \sim 0.4 \mathrm{~ns}$. For QD3, Figure $\mathrm{S} 2$ in SM provides 18 combination of polarization-resolved $\mathrm{XX}-\mathrm{X}$ correlations; the $\mathrm{AA} / \mathrm{AD}$ and RL/RR bases show nearly zero contrast due to independent $\mathrm{HH}$ and $\mathrm{VV}$ emissions. The lh-hh mixing and EIP lead to unbalanced bunching peaks in the DA/AD or HV/VH basis. The deduced $\mathrm{F}$ is an optimal one excluding the unbalanced bunching and without background subtraction. Due to a finite lh-hh mixing $(\beta \leq 0.2)$, the two split lines HX and LX are nearly orthogonal linearly polarized $[22,23]$ and can be purely filtered. Figure 7 plots these $F$ we measured and the theoretical (F, FSS) curves (i.e., Equation 1 presented below, where $f$ is the fidelity, $g_{H V^{\prime}}$ the fraction of photons uninfluenced by spin scattering, $x$ and $k$ the proportion of light exclusively emitted by the QD defined by $T_{1}, T_{\mathrm{ss}}$ and $g^{2}(0)$ given in the inset) [2,3]. QD6, QD3 and QD23 with $\mathrm{T}_{1} \sim 0.3 \mathrm{~ns}$ showed a reduced $\mathrm{F}$ from spin flip compared to the fitting curve with $\mathrm{T}_{\mathrm{ss}}=1.9 \mathrm{~ns}$; $\mathrm{QD} 8$ with $\mathrm{T}_{1} \sim 0.4 \mathrm{~ns}$ and weak $\mathrm{XX}$ (i.e., few electrons in the barrier for spin flip) showed a higher $\mathrm{F}, 0.57$, little varied from the fitting curve prediction with $\mathrm{T}_{\mathrm{ss}}=100 \mathrm{~ns}$, reflecting little spin flip or spin scattering. A higher electric field to deplete these electrons as QDs in Figure 3 with AlGaAs barrier as capping [18,25-27] to reduce tunneling and a sub-bandgap optical excitation is desirable.

$$
\begin{gathered}
f=\left(1+g_{H V}^{\prime}+2 k g_{H V}^{\prime} /\left(1+x^{2}\right)\right) / 4 \\
g_{H V}^{\prime}=\left(1+T_{1} / T_{S S}\right)^{-1}, x=\mathrm{FSS} \times g_{H V}^{\prime} T_{1} / \hbar, k=1-g^{2}(0)
\end{gathered}
$$

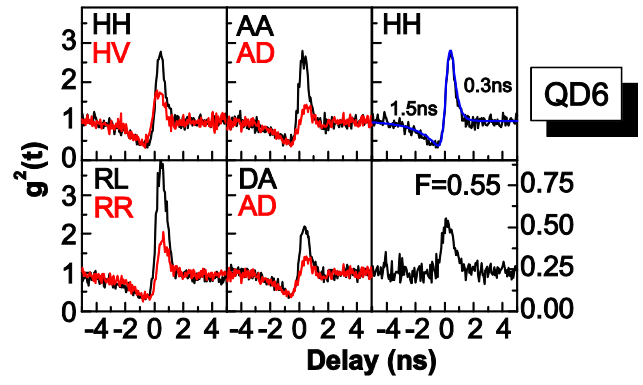

(a)

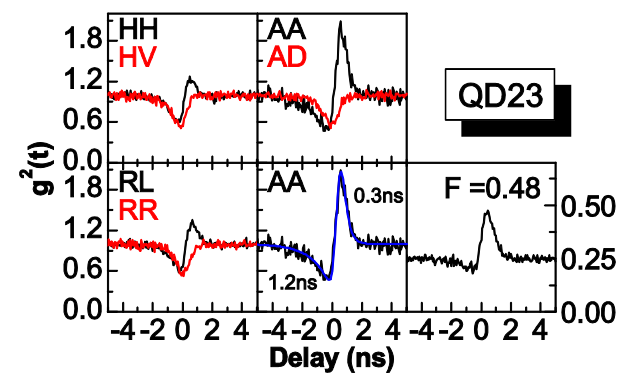

(c)

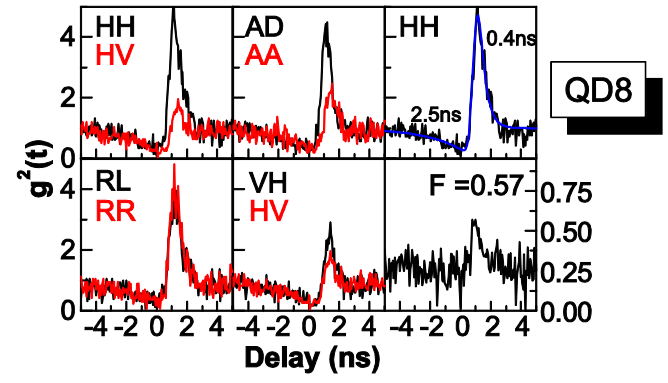

(b)

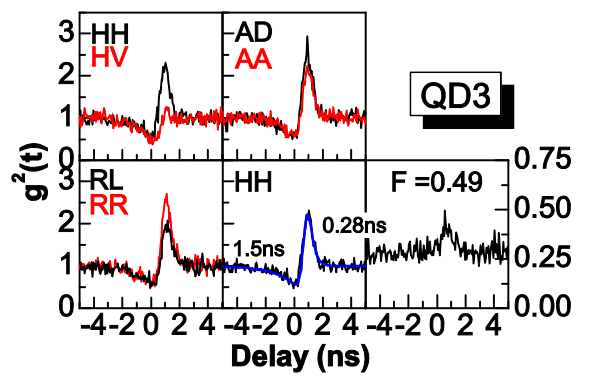

(d)

Figure 6. Polarization-resolved XX-X cross-correlations in (a) QD6, (b) QD8, (c) QD23, and (d) QD3. The estimated F, the convoluted fitting (blue) and the fitting times are given. 


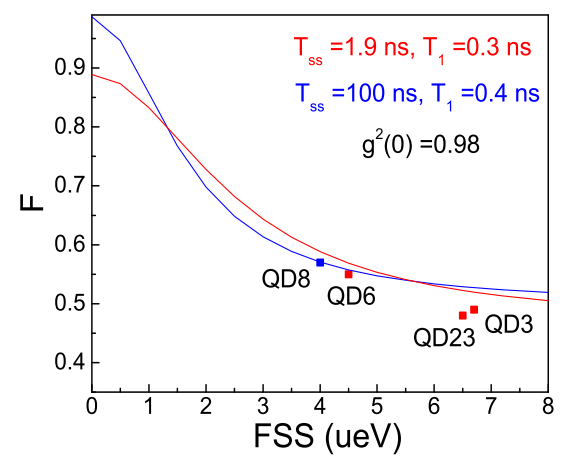

Figure 7. Experimental F of QDs we measured (QD3, QD8, QD6 and QD23) and theoretical (F, FSS) curves with $\mathrm{g}^{2}(0), \mathrm{T}_{\mathrm{ss}}$ and $\mathrm{T}_{1}$ given.

\section{Conclusions}

In conclusion, with a Si dopant above an (001)-based InAs/GaAs QD to build a local electric field, $\mathrm{D}_{3 \mathrm{~h}}$ symmetric excitons and small FSS $\sim 4 \mu \mathrm{eV}$ (with a possible zero minimum) are obtained, which facilitates the FSS erasure with a little junction field tuning. The diverse field projection in the lateral plane shows various FSS, LX and EIP with a universal field dependence; a strong vertical field is desired to restore $\mathrm{X} 1-\mathrm{X} 2$ degeneracy with no EIP. With FSS $\sim 4 \mu \mathrm{eV}$ and $\mathrm{T}_{1} \sim 0.3 \mathrm{~ns}$ in a DBR cavity, polarization-resolved $\mathrm{XX}-\mathrm{X}$ correlations reflect cascade emission with a fidelity $\sim 0.55$ to the maximal entangled state $|\mathrm{HH}\rangle+|\mathrm{VV}\rangle$; spin flip from electrons in the barrier can be reduced with a weak XX excitation or in a higher electric field with these electrons depleted, combined with a sub-bandgap excitation. The breaking of wavefunction degeneracy with a lateral field along [1-10] ensures independent $\mathrm{HH}$ and VV cascade emission with robust polarization correlation and no spin flip, promising for photon-pair correlation applications.

Supplementary Materials: The following are available online at https: / www.mdpi.com/article / 10.3390/cryst11101194/s1, Figure S1: Excitation power-dependent $\mu$ PL spectra in QD5 with more $D_{3 h}$ exciton emission features and QD4 with similar $D_{3 h}$ exciton emission features as QD1 and FSS $\sim 11 \mu \mathrm{eV}$, Figure S2: Eighteen combinations of polarization-related XX-X correlations in QD3 $(\mathrm{FSS}=6.8 \mu \mathrm{eV}, \mathrm{F}=0.49)$, Figure S3: $\mu$ PL spectrum, FSS oscillation and EIP in a polar plot in QD19.

Author Contributions: Conceptualization of QD structure, Z.N., X.S. (Xingjun Shang) and B.M.; methodology for QD growth and optical measurements, X.S. (Xingjun Shang), S.L., H.L., B.M., X.S. (Xiangbin Su) and H.N.; formal analysis, X.S. (Xiangbin Su), Y.C., J.S., H.H., X.D., Y.J., B.S., B.L. and Z.N.; investigation, X.S. (Xingjun Shang), S.L. and H.L.; validation, X.S. (Xingjun Shang), S.L., X.D.; resources, X.S. (Xingjun Shang); data curation, S.L., H.L. and Z.N.; writing-original draft preparation, X.S. (Xingjun Shang); writing-review and editing, X.S. (Xingjun Shang), S.L. and Z.N.; visualization, X.S. (Xingjun Shang); supervision, Z.N.; project administration, Z.N.; funding acquisition, Z.N. All authors have read and agreed to the published version of the manuscript.

Funding: This research was funded by the National Key Technologies R\&D Program of China, Grant No. 2018YFA0306100), the National Natural Science Foundation of China (Grant No. 61505196), the Program of Beijing Academy of Quantum Information Sciences (Grant No. Y18G01), and the Scientific Instrument Developing Project of Chinese Academy of Sciences (Grant No. YJKYYQ20170032).

Data Availability Statement: The data that support the findings of this study are available from the corresponding author upon reasonable request.

Conflicts of Interest: The authors declare no conflict of interest. 


\section{References}

1. Keil, R.; Zopf, M.; Chen, Y.; Höfer, B.; Zhang, J.; Ding, F.; Schmidt, O.G. Solid-state ensemble of highly entangled photon sources at rubidium atomic transitions. Nat. Commun. 2017, 8, 15501. [CrossRef]

2. Liu, J.; Su, R.; Wei, Y.; Yao, B.; Da Silva, S.F.C.; Yu, Y.; Iles-Smith, J.; Srinivasan, K.; Rastelli, A.; Li, J.; et al. A solidstate source of strongly entangled photon pairs with high brightness and indistinguishability. Nat. Nanotechnol. 2019, 14, 586-593. [CrossRef] [PubMed]

3. Hudson, A.J.; Stevenson, R.M.; Bennett, A.J.; Young, R.J.; Nicoll, C.A.; Atkinson, P.; Cooper, K.; Ritchie, D.A.; Shields, A.J. Coherence of an Entangled Exciton-Photon State. Phys. Rev. Lett. 2007, 99, 266802. [CrossRef] [PubMed]

4. Zhang, J.; Wildmann, J.S.; Ding, F.; Trotta, R.; Huo, Y.; Zallo, E.; Huber, D.; Rastelli, A.; Schmidt, O.G. High yield and ultrafast sources of electrically triggered entangled-photon pairs based on strain-tunable quantum dots. Nat. Commun. 2015, 6, 10067. [CrossRef] [PubMed]

5. $\quad$ Chen, Z.-S.; Ma, B.; Shang, X.-J.; He, Y.; Zhang, L.-C.; Ni, H.-Q.; Wang, J.-L.; Niu, Z.-C. Telecommunication Wavelength-Band Single-Photon Emission from Single Large InAs Quantum Dots Nucleated on Low-Density Seed Quantum Dots. Nanoscale Res. Lett. 2016, 11, 382. [CrossRef]

6. Tang, J.-S.; Zhou, Z.-Q.; Wang, Y.-T.; Li, Y.-L.; Liu, X.; Hua, Y.-L.; Zou, Y.; Wang, S.; He, D.-Y.; Chen, G.; et al. Storage of multiple single-photon pulses emitted from a quantum dot in a solid-state quantum memory. Nat. Commun. 2015, 6, 8652. [CrossRef]

7. Birowosuto, M.D.; Sumikura, H.; Matsuo, S.; Taniyama, H.; Van Veldhoven, P.J.; Nötzel, R.; Notomi, M. Fast Purcell-enhanced single photon source in 1,550-nm telecom band from a resonant quantum dot-cavity coupling. Sci. Rep. 2012, 2, 321. [CrossRef]

8. Sapienza, L.; Malein, R.N.; Kuklewicz, C.E.; Kremer, P.E.; Srinivasan, K.; Griffiths, A.; Clarke, E.; Gong, M.; Warburton, R.J.; Gerardot, B.D. Exciton fine-structure splitting of telecom-wavelength single quantum dots: Statistics and external strain tuning. Phys. Rev. B 2013, 88, 155330. [CrossRef]

9. Seidl, S.; Gerardot, B.; Dalgarno, P.; Kowalik, K.; Holleitner, A.W.; Petroff, P.M.; Karrai, K.; Warburton, R.J. Statistics of quantum dot exciton fine structure splittings and their polarization orientations. Phys. E Low-Dimens. Syst. Nanostruct. 2008, 40, 2153-2155. [CrossRef]

10. Su, D.; Dou, X.; Wu, X.; Liao, Y.; Zhou, P.; Ding, K.; Ni, H.; Niu, Z.; Zhu, H.; Jiang, D.; et al. Tuning exciton energy and fine-structure splitting in single InAs quantum dots by applying uniaxial stress. AIP Adv. 2016, 6, 045204. [CrossRef]

11. Chen, H.; Zhuo, Z.; Huang, J.; Dou, X.; He, X.; Ding, K.; Ni, H.; Niu, Z.; Jiang, D.; Sun, B. Correlation between exciton polarized lifetime and fine structure splitting in InAs/GaAs quantum dots. Appl. Phys. Lett. 2020, 116, 082101. [CrossRef]

12. Seguin, R.; Schliwa, A.; Germann, T.D.; Rodt, S.; Potschke, K.; Pohl, U.W.; Bimberg, D.; Winkelnkemper, M.; Hammerschmidt, T.; Kratzer, P. Control of fine-structure splitting and excitonic binding energies in selected individual InAs/GaAs quantum dots. Appl. Phys. Lett. 2006, 89, 263109. [CrossRef]

13. Treu, J.; Schneider, C.; Huggenberger, A.; Braun, T.; Reitzenstein, S.; Höfling, S.; Kamp, M. Substrate orientation dependent fine structure splitting of symmetric In(Ga)As/GaAs quantum dots. Appl. Phys. Lett. 2012, 101, 022102. [CrossRef]

14. Juska, G.; Dimastrodonato, V.; Mereni, L.O.; Gocalinska, A.; Pelucchi, E. Towards quantum-dot arrays of entangled photon emitters. Nat. Photon. 2013, 7, 527-531. [CrossRef]

15. Mereni, L.O.; Marquardt, O.; Juska, G.; Dimastrodonato, V.; O’Reilly, E.P.; Pelucchi, E. Fine-structure splitting in large-pitch pyramidal quantum dots. Phys. Rev. B 2012, 85, 155453. [CrossRef]

16. Karlsson, K.F.; Oberli, D.A.; Dupertuis, M.; Troncale, V.; Byszewski, M.; Pelucchi, E.; Rudra, A.; Holtz, P.O.; Kapon, E. Spectral signatures of high-symmetry quantum dots and effects of symmetry breaking. New J. Phys. 2015, 17, 103017. [CrossRef]

17. Dupertuis, M.A.; Karlsson, F.; Oberli, D.; Pelucchi, E.; Rudra, A.; Holtz, P.O.; Kapon, E. Symmetries and the Polarized Optical Spectra of Exciton Complexes in Quantum Dots. Phys. Rev. Lett. 2011, 107, 127403. [CrossRef] [PubMed]

18. Bennett, A.J.; Pooley, M.A.; Stevenson, R.M.; Ward, M.B.; Patel, R.B.; De La Giroday, A.B.; Sköld, N.; Farrer, I.; Nicoll, C.A.; Ritchie, D.A.; et al. Electric-field-induced coherent coupling of the exciton states in a single quantum dot. Nat. Phys. 2010, 6, 947-950. [CrossRef]

19. Trotta, R.; Zallo, E.; Ortix, C.; Atkinson, P.; Plumhof, J.D.; Brink, J.V.D.; Rastelli, A.; Schmidt, O.G. Universal Recovery of the Energy-Level Degeneracy of Bright Excitons in InGaAs Quantum Dots without a Structure Symmetry. Phys. Rev. Lett. 2012, 109, 147401. [CrossRef]

20. Huber, D.; Reindl, M.; da Silva, S.F.C.; Schimpf, C.; Martín-Sánchez, J.; Huang, H.; Piredda, G.; Edlinger, J.; Rastelli, A.; Trotta, R. Strain-Tunable GaAs Quantum Dot: A Nearly Dephasing-Free Source of Entangled Photon Pairs on Demand. Phys. Rev. Lett. 2018, 121, 033902. [CrossRef]

21. Chen, Y.; Zhang, J.; Zopf, M.; Jung, K.; Zhang, Y.; Keil, R.; Ding, F.; Schmidt, O.G. Wavelength-tunable entangled photons from silicon-integrated III-V. quantum dots. Nat. Commun. 2015, 7, 10387. [CrossRef]

22. Belhadj, T.; Amand, T.; Kunold, A.; Simon, C.-M.; Kuroda, T.; Abbarchi, M.; Mano, T.; Sakoda, K.; Kunz, S.; Marie, X.; et al. Impact of heavy hole-light hole coupling on optical selection rules in GaAs quantum dots. Appl. Phys. Lett. 2010, 97, 051111. [CrossRef]

23. Tonin, C.; Hostein, R.; Voliotis, V.; Grousson, R.; Lemaitre, A.; Martinez, A. Polarization properties of excitonic qubits in single self-assembled quantum dots. Phys. Rev. B 2012, 85, 155303. [CrossRef]

24. Abbarchi, M.; Mano, T.; Kuroda, T.; Ohtake, A.; Sakoda, K. Polarization Anisotropies in Strain-Free, Asymmetric, and Symmetric Quantum Dots Grown by Droplet Epitaxy. Nanomaterials 2021, 11, 443. [CrossRef] [PubMed] 
25. Wei, G.; Forrest, S.R. Intermediate-Band Solar Cells Employing Quantum Dots Embedded in an Energy Fence Barrier. Nano Lett. 2007, 7, 218. [CrossRef] [PubMed]

26. Varghese, A.; Yakimov, M.; Tokranov, V.; Mitin, V.; Sablon, K.; Sergeev, A.; Oktyabrsky, S. Complete voltage recovery in quantum dot solar cells due to suppression of electron capture. Nanoscale 2016, 8, 7248. [CrossRef]

27. Sablon, K.A.; Little, J.W.; Olver, K.A.; Wang, Z.M.; Dorogan, V.G.; Mazur, Y.I.; Salamo, G.J.; Towner, F.J. Effects of AlGaAs energy barriers on InAs/GaAs quantum dot solar cell. J. Appl. Phys. 2010, 108, 074305. [CrossRef]

28. Li, M.F.; Yu, Y.; He, J.F.; Wang, L.J.; Zhu, Y.; Shang, X.J.; Ni, H.Q.; Niu, Z.C. In situ accurate control of 2D-3D transition parameters for growth of low-density InAs/GaAs self-assembled quantum dots. Nanoscale Res. Lett. 2013, 8, 86. [CrossRef]

29. Shang, X.-J.; Xu, J.-X.; Ma, B.; Chen, Z.-S.; Wei, S.-H.; Li, M.-F.; Zha, G.-W.; Zhang, L.-C.; Yu, Y.; Ni, H.-Q.; et al. Proper In deposition amount for on-demand epitaxy of InAs/GaAs single quantum dots. Chin. Phys. B 2016, 25, 107805. [CrossRef]

30. Ma, B.; Chen, Z.S.; Wei, S.H.; Shang, X.J.; Ni, H.Q.; Niu, Z.C. Single photon extraction from self-assembled quantum dots via stable fiber array coupling. Appl. Phys. Lett. 2017, 110, 142104. [CrossRef]

31. Yu, Y.; Shang, X.-J.; Li, M.-F.; Zha, G.-W.; Xu, J.-X.; Wang, L.-J.; Wang, G.-W.; Ni, H.-Q.; Dou, X.-M.; Sun, B.-Q.; et al. Single InAs quantum dot coupled to different "environments" in one wafer for quantum photonics. Appl. Phys. Lett. 2013, $102,201103$.

32. Li, S.L.; Shang, X.J.; Chen, Y.; Su, X.; Hao, H.; Liu, H.; Zhang, Y.; Ni, H.Q.; Niu, Z.C. Wet-etched microlens array for 200 nm spatial isolation of epitaxial single QDs and $80 \mathrm{~nm}$ broadband enhancement of their quantum light extraction. Nanomaterials 2021, 11, 1136. [CrossRef]

33. Shang, X.-J.; Ma, B.; Ni, H.Q.; Chen, Z.S.; Li, S.L.; Chen, Y.; He, X.; Su, X.; Shi, Y.; Niu, Z.-C. C2v and D3h symmetric InAs quantum dots on GaAs (001) substrate: Exciton emission and a defect field influence. AIP Adv. 2020, 10, 085126. [CrossRef]

34. He, L.; Gong, M.; Li, C.F.; Guo, G.C.; Zunger, A. Highly Reduced Fine-Structure Splitting in InAs/InP Quantum Dots Offering an Efficient On-Demand Entangled 1.55-um Photon Emitter. Phys. Rev. Lett. 2008, 101, 157405. [CrossRef] [PubMed]

35. Nguyen, H.S.; Sallen, G.; Abbarchi, M.; Ferreira, R.; Voisin, C.; Roussignol, P.; Cassabois, G.; Diederichs, C. Photoneutralization and slow capture of carriers in quantum dots probed by resonant excitation spectroscopy. Phys. Rev. B 2013, 87, 115305. [CrossRef]

36. Ramírez, H.Y.; Chou, Y.-L.; Cheng, S.-J. Effects of electrostatic environment on the electrically triggered production of entangled photon pairs from droplet epitaxial quantum dots. Sci. Rep. 2019, 9. [CrossRef]

37. Ghali, M.; Ohtani, K.; Ohno, Y.; Ohno, H. Generation and control of polarization-entangled photons from GaAs island quantum dots by an electric field. Nat. Commun. 2012, 3, 661. [CrossRef] [PubMed] 